\title{
Improving Chronic Kidney Disease Care in Primary Care Practices: An Upstate New York Practice- based Research Network (UNYNET) Study
}

\author{
Chester H. Fox, MD, Andrew Swanson, BA, Linda S. Kahn, PhD, \\ Katheryn Glaser, BA, and Brian M. Murray, MD
}

Background: With the prevalence of chronic kidney disease (CKD) in the United States rising from 10\% to $13 \%$, implementation of the evidence-based Kidney Disease Outcomes Quality Initiative guidelines, which were developed for the delay of progression of CKD, is of increasing importance in primary care offices. Previous studies have shown limited knowledge and uptake of Kidney Disease Outcomes Quality Initiative guidelines by primary care physicians. CKD and its complications are still largely under-diagnosed and under-treated. A multifaceted quality improvement study was undertaken to test if these guidelines could be implemented to improve CKD care in underserved practices.

Methods: Using a combination of practice enhancement assistants, computer decision-making support, and academic detailing, we sought to increase physician awareness and care of CKD in 2 inner-city practices. Using these 3 modalities, a rapid-cycle quality improvement process was implemented.

Results: One hundred eighty-one patients met the inclusion criteria of having a glomerular filtration rate $<60$. This represented a 100\% sample of patients with CKD at baseline. Recognition of CKD improved significantly from $30(21 \%)$ to $114(79 \%)(P<.001)$. Diagnosis of anemia also increased significantly from $26(33 \%)$ to $53(67 \%)(P<.001)$. Angiotensin-converting enzyme inhibitor and aspirin use did not change significantly $(P=.31$ and $P=.233$, respectively $)$. Changes in medications that did show significance were metformin use, which decreased $50 \%$ from 12 to 6 patients $(P<.001)$, and nonsteroidal anti-inflammatory drug use, which decreased $41 \%$ from 23 to 14 patients $(P<.001)$. Mean glomerular riltration rate increased significantly from 45.75 to $47.34(P<.001)$.

Discussion: Recognition and treatment of CKD and its complications can be markedly improved in primary care offices using a combination of practice enhancement assistants, computer decision-making support, and academic detailing. A significant rise in glomerular riltration rate, although small, was a surprising and encouraging result. Larger studies in a more geographically spread region are needed to confirm these preliminary results. (J Am Board Fam Med 2008;21:522-30.)

The prevalence of chronic kidney disease (CKD) in the United States has increased over the past 10 years, from $10 \%$ during 1988 to $1994^{1}$ to $13.1 \%$ during 1999 to $2004 .^{2}$ Despite this increasing prevalence, awareness of CKD among the population has been relatively low. ${ }^{1,3}$ Risk factors for CKD include increased age, hypertension, diabetes, car-

This article was externally peer reviewed.

Submitted 15 February 2008; revised 11 July 2008; accepted 15 July 2008.

From the Department of Family Medicine (CHF, AS, LSK, KG, BMM) and the Division of Nephrology (BMM), School of Medicine and Biomedical Sciences, State University of New York at Buffalo.

Funding: Grant provided by the Agency for Health Research and Quality Grant Number: AHRQ R03 H5016031. Conflict of interest: none declared.

Corresponding author: Chester H. Fox, Family Medicine, University at Buffalo, 462 Grider St, Buffalo, New York 14215 (E-mail: chetfox@gmail.com). diovascular disease, and a family history of kidney disease $^{4-6}$; thus the number of individuals at risk for $\mathrm{CKD}$ will continue to rise. ${ }^{7} \mathrm{CKD}$ disproportionately affects African-Americans and other minority populations. ${ }^{8}$ Having CKD increases the likelihood of suffering a myocardial infarction and decreases the chance of survival. ${ }^{9,10}$ Early recognition, proper management, and early referral have the greatest effect on slowing the progression of CKD. ${ }^{11}$ The best way to diagnose early CKD is through the use of 2 tests: the estimated glomerular filtration rate $(\mathrm{GFR})^{12}$ and the urine albumin/creatinine ratio. ${ }^{13}$

Evidence-based guidelines demonstrate that the following have been effective in slowing the progression of $\mathrm{CKD}^{14-18}$ : early recognition of $\mathrm{CKD}$; better treatment of hypertension, diabetes, hyperlipidemia, anemia, and abnormal bone mineral me- 
tabolism laboratory work-ups; discontinuation of nonsteroidal anti-inflammatory drugs (NSAIDs); the use of aspirin and angiotensin-converting enzyme (ACE) inhibitors or angiotensin receptor blockers (ARBs).

The guidelines for slowing the progression of CKD, which are embodied in the National Kidney Foundations Kidney Disease Outcomes Quality Initiative (KDOQI) ${ }^{19}$ have had limited uptake nationally. ${ }^{4,20}$ A lack of awareness, their relative newness, and the length of the guideline documents ${ }^{14}$ are possible barriers to physician use of these guidelines. The National Institutes of Health has recognized that implementation of evidence-based research findings has been problematic. Two recent studies of primary care physician's (PCP) offices have demonstrated that a large number of physicians were not aware of the KDOQI guidelines. ${ }^{14,20}$

To our knowledge, this is the first study to test the implementation of these evidence-based CKD guidelines in PCP practices. A literature search of Medline from 1996 to present using the keywords "KDOQI" and "quality improvement" yielded 43 references, none of which were interventional studies.

Practice facilitation using practice enhancement assistants (PEAs) has been shown to improve care. ${ }^{15,16}$ A PEA assists primary care practices in research and quality improvement activities, providing a framework for translating research into practice. ${ }^{16,17}$ PEAs usually have nursing degrees or master's degrees in health sciences or related fields and function as both a research assistant and a quality improvement (QI) agent for clinics that may not have enough practice staff or resources to pursue research initiatives or implement QI projects. As a research assistant, the PEA is responsible for the data collection aspects of a research project and may spend a half a day per week or more at each site within a group of practices. As a quality improvement agent, the PEA develops a relationship with a group of practices over time to help evaluate and improve the quality of care. They will initiate a quality improvement project that the staff can eventually continue. PEAs build relationships, facilitate change, implement national guidelines, and share best practices within a practice-based research network.

Computer decision-making support ${ }^{17,18}$ and academic detailing ${ }^{21,22}$ have also been shown to be effective in improving care. Therefore, a multimodal QI study that included PEAs, computer decision-making support, and academic detailing designed to increase implementation of evidencebased CKD guidelines in 2 underserved urban minority practices was completed. This article presents the preliminary improvement data from the 2 practice intervention sites.

\section{Methods}

\section{Patients and Setting}

This QI intervention was accomplished in 2 underserved primary care practices. The first site is a private family medicine practice providing comprehensive medical services to an underserved urban neighborhood with a high proportion of international refugees. There are $3 \mathrm{MDs}$ and 2 nurse practitioners on staff and approximately 5000 active medical records. This center has a fully implemented electronic medical records (EMR) system. The second site is urban and predominantly African-American, with approximately 7000 patients and a paper charting system. This clinic's staff consists of 3 physicians and 2 nurse practitioners.

\section{Practice Enbancement Assistants}

Two PEAs were used in this QI project to provide assistance to the practicing physicians and to implement computer-guided support systems. The PEAs had a "business associate's agreement" with each of the practices. The State University of New York at Buffalo Health Sciences Institutional Review Board granted human subjects approval for the study. The initial chart audit selected patients with diabetes mellitus and/or hypertension to identify patients with a GFR $<60 \mathrm{~mL} / \mathrm{min} / 1.73 \mathrm{~m}^{2}$ All patients with GFR $<60 \mathrm{~mL} / \mathrm{min} / 1.73 \mathrm{~m}^{2}$ were included, regardless of diagnosis or disease stage. A CKD patient registry was then created and given to each provider and the office staff. Based on initial chart audit, recommendations promoting the implementation of national guidelines were used to begin the QI cycle.

In accordance with the KDOQI guidelines, PEAs facilitated the use of a computer decisionmaking support program to create recommendations for each provider on a patient-specific basis. Patient registries were created for intervention sites and were given to office staff and individual providers. For the site using an EMR system, a task 
reminder was placed in the physician "to do" section and in the patient EMR. The physician completed the task in the medical record. A paper version of the recommendation was also left for providers to accept, reject, or modify. Once approved, reminder notes were put into patient charts to diagnose CKD and/or anemia, discontinue harmful medications, request additional laboratory work-ups and/or referrals, intensify chronic disease management, and treat CKD complications. The physician responded to these reminders in the EMR. For the site using paper charts, a modified version of the recommendation, in the form of a letter, was left in patients' charts to facilitate the changes during patient visits. Patients who had not been seen in 6 months were contacted by phone and encouraged to make an appointment. After 12 weeks, PEAs conducted a second chart review and a second recommendation was made based on any changes or unimplemented recommendations. This cycle continued until a 1-year intervention was completed in both sites.

\section{Computer Decision-Making Support}

A computer decision-making support system, the PEA Work Tool (Appendix 1), was created based on the KDOQI guidelines. This work tool extracted a limited data set necessary for the implementation these guidelines. These clinical elements included current GFR; current Hba1c; current hemoglobin; medications associated with treatment of $\mathrm{CKD}$; and bone mineral laboratory tests of calcium, phosphorous, intact parathyroid hormone, and $25-\mathrm{OH}$ vitamin $\mathrm{D}$ levels, which are associated with complications of CKD.

After the extraction of these chart elements, a recommendation reminder sheet was created for each PCP. This reminder sheet contained the current status of the patient and recommendations for follow-up based on the guidelines. The Appendix 1 shows the reminder sheet as it would be seen by the PCP during everyday use. Every 4 weeks, academic detailing was undertaken. The principal investigator (CF), a practicing physician, would meet with the physicians at each site to discuss the recommendations and follow-up. A 3-month cyclical chart review was implemented to check for QI in the implementation of these recommendations (Figure 1).

\section{Academic Detailing}

Academic detailing was accomplished with a monthly luncheon with each of the sites and the PI. After the data presentations, issues of implementation were discussed. The clinicians used this time to learn about the importance of the guidelines and to discuss facilitating factors and barriers.

\section{Case Finding}

In the practice with the EMR, the system was queried to identify all patients with a GFR $<60$ $\mathrm{mL} / \mathrm{min} / 1.73 \mathrm{~m}^{2}$. Patients older than 18 years of age who had been with the practice for at least 1 year were all included in the study. In the practice that used paper charts, the practice management system was queried and all patients who had a diagnosis of either hypertension or diabetes had their charts reviewed. All patients who had a GFR $<60 \mathrm{~mL} / \mathrm{min} / 1.73 \mathrm{~m}^{2}$ and had been members of the practice for 1 year were included in the study.

\section{Data Analysis}

After the extraction of the clinical elements, data were exported from Microsoft Access (Microsoft, Redmond, WA) into the Statistical Package for the Social Sciences software (SPSS 11.5, Chicago, IL) for analysis. Descriptive statistics were performed on laboratory scores, medication management, and disease recognition. Fisher's exact tests were conducted on dichotomous variables (eg, current medications and current diagnoses) comparing baseline rates to rates after intervention. Paired sample $t$ tests were conducted on GFR levels to test for a significant change.

\section{Results}

One hundred eighty-one patients met the inclusion criteria. Seventy-seven patients in the practice using EMRs were identified as a result of a data query. In the practice with paper charts, approximately 600 charts of patients with hypertension or diabetes were reviewed and $104 \mathrm{pa}$ tients who had a GFR $<60 \mathrm{~mL} / \mathrm{min} / 1.73 \mathrm{~m}^{2}$ were identified. All of these cases were identified by examining the laboratory data section of the chart. Demographics of the 2 practices are summarized in Table 1.

The results of the study are summarized in Table 2. Recognition of CKD, defined by having a CKD diagnosis on either the billing information, 


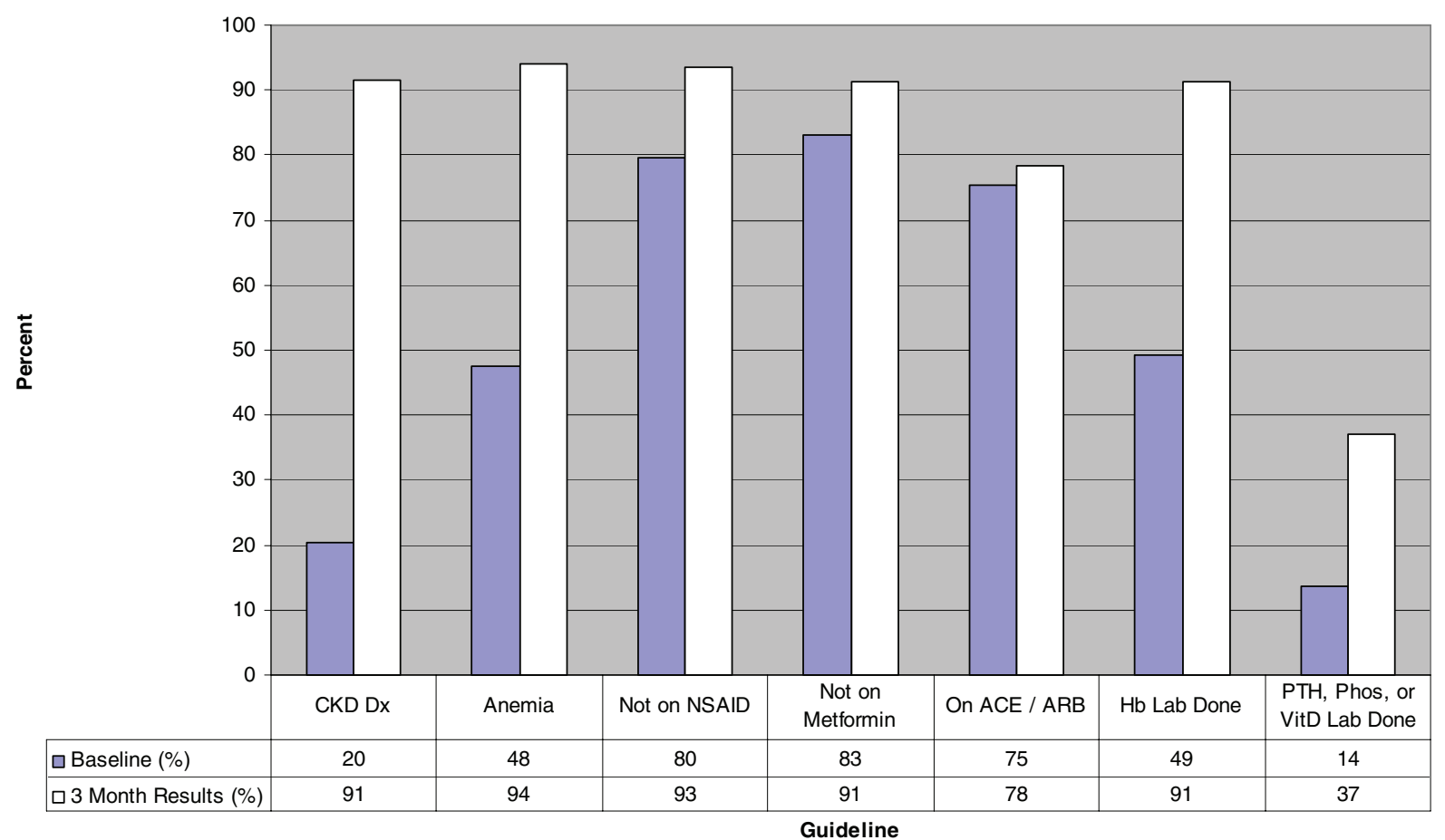

Figure 1. Quarter 1 results. Change in performance of implementing CKD evidence-based guidelines for a 3-month period. Dx, diagnosis; NSAID, nonsteroidal anti-inflammatory drug; ACE, angiotensin-converting enzyme inhibitor; ARB, angiotensin receptor blocker; Hb, hemoglobin; PTH, parathyroid hormone; Phos, phosphate; Vit D, vitamin D.

the problem list, or the progress notes, was very low at $21 \%$. Recognition of anemia, defined as having a hemoglobin level of $<12 \mathrm{mg} / \mathrm{dL}$ for either men or women, was also low at $33 \%$. These scores improved with intervention to $79 \%(P<.001)$ and $67 \%(P<.001)$, respectively.

Use of metformin and NSAID medications decreased after the intervention. Metformin use was decreased by $50 \%$ during the intervention $(P<$ .001). NSAID use also showed a significant reduction of $41 \%(P<.001)$. Medications associated with positive changes in CKD (aspirin and ACE

Table 1. Description of Chronic Kidney Disease Patient Participants

\begin{tabular}{lcc}
\hline & Site 1 (\%) & Site 2 (\%) \\
\hline On Medicaid (any form) (\%) & 62 & 49.5 \\
Without health insurance (\%) & 10 & 12.6 \\
Race/ethnicity (\%) & & \\
African-American & 30 & 95 \\
Hispanic & 35 & $<1$ \\
Asian or Pacific Islander & 10 & $<1$ \\
White & 25 & 5 \\
Urban patients (\%) & 85 & 96 \\
\hline
\end{tabular}

inhibitor/ARB) showed small changes that were not statistically significant. Mean GFR levels increased significantly by $1.59 \mathrm{~mL} / \mathrm{min}(P<.001)$.

\section{Discussion}

Our findings support previous reports in the literature that recognition of CKD and its complications are not well recognized in primary care practices. ${ }^{14,20}$ The use of a multifaceted intervention with PEAs, computer decision-making support, and academic detailing resulted in statistically and clinically significant improvements in the recognition of CKD and anemia. In addition, the use of potentially harmful medicines, specifically NSAIDs and metformin, was significantly decreased. The use of helpful medicines (aspirin and ACE inhibitiors/ARBs) was varied, with a nonsignificant increase in aspirin use and a nonsignificant decrease in ACE inhibitor/ARB use.

Mean GFR increased a statistically significant $1.59 \mathrm{~mL} / \mathrm{min}$. This is an encouraging finding because it suggests the intriguing possibility that CKD in its early stages may be reversible. It is difficult to know if this change is clinically signifi- 


\begin{tabular}{lcrr}
\hline & At Baseline (n [\%]) & After Intervention (n [\%]) & $P$ \\
\hline CKD diagnosis & $30(21)$ & $114(79)$ & $<.001$ \\
Anemia diagnosis & $26(33)$ & $53(67)$ & $<.001$ \\
Aspirin use & $41(30)$ & $48(35)$ & .233 \\
Metformin use & $17(12)$ & $8(6)$ & $<.001$ \\
NSAID use & $23(17)$ & $14(10)$ & $<.001$ \\
ACE inhibitor/ARB use & $84(62)$ & $79(58)$ & .31 \\
GFR (mean [SD]) & $45.75(15.67)$ & $47.34(14.29)$ & $<.001$ \\
\hline
\end{tabular}

CKD, chronic kidney disease; NSAID, nonsteroidal anti-inflammatory drug; ACE, angiotensin-converting enzyme; ARB, angiotensin receptor blocker; GFR, glomerular filtration rate.

cant because of the small size and short duration of this study.

The use of the PEAs was essential because they facilitated the recommendation process and started the QI cycle. PEAs were readily accepted by the practice staff. PEAs were asked to do many things at the sites and both sites are eager to work in other projects in which a PEA is involved. The practices requested that a point of care summary guide to treat CKD be developed to help the clinician. A 2-page bulleted guide was completed and distributed to the clinicians (Appendix 2). The clinicians responded positively and then requested a companion patient guide that could be given to the patients with the same information. This was brought back to the practices by the PEAs and it resulted in a request that the patient guide be translated into Spanish and Arabic. This is in the process of being completed. In addition, the site with a large number of refugees requested pictorial guides to teach their patients about hospitals and pharmacies and their locations. The PEAs put these together and the practices were very pleased with them.

Computer decision-making support was also well received by the practices but, as would be expected, it was much more challenging in the office using paper records. On average, it took 4 weeks longer to implement a change in practice in the paper-based office and required considerably more effort on the part of the PEAs. Having paper charts found and pulled for audits was a significant barrier that was not present in the practice with the EMRs. At the request of the providers the practice using EMRs used both task lists and chart reminders to implement the guidelines, whereas the paper office chose to implement only chart reminders. The providers in the paper practice instructed the
PEAs as to how they wanted this work flow to occur and stated they did not want flowsheets or task lists.

In response to this form of academic detailing, all clinicians reported a greater awareness of CKD when they were seeing their patients. The clinicians expressed the need to understand why the guidelines were important before implementation. Problems with the study were also addressed during these academic detailing visits. For example, one clinician was reluctant to implement the guidelines because he was afraid that he would be profiled by a health plan for doing too many laboratory tests and would be sanctioned. He was instructed in the proper use of diagnosis codes on the laboratory slips so as to clinically justify the tests. This resolved the issue.

We found that physicians' lack of awareness regarding the KDOQI guidelines was apparent at baseline by the low rates of diagnosis and the lack of appropriate treatment (bone mineral laboratory tests, medication use, etc). However, lack of awareness may not be the only underlying factor for these problems. PCPs are confronted with a limited time to deliver appropriate and recommended chronic disease management, in addition to diagnosing new problems and providing preventive care. ${ }^{23-26}$ In addition, because of the overlap between diabetes, cardiovascular disease, and CKD, it is possible that physicians prioritize treatment for diabetes or cardiovascular disease without realizing the underlying pathophysiologic link between these diseases and $\mathrm{CKD} .^{27}$

Our study demonstrates that a multimodal approach to QI using a combination of computer decision-making support, academic detailing, and PEAs can improve the recognition and evidence-based management of CKD. The fact that these improve- 
ments occurred in underserved practices is encouraging, especially because many of the patients have significant challenges to improving their health such as transportation, health literacy, cultural issues, and not having insurance or being under-insured.

This study has a number of limitations. These include the relatively small sample size, the lack of geographic variability and the short duration of the study. The study design before and after intervention can create an undetectable cointervention bias. The 2 practices show similarities in demographics and are both in the same major city. Larger and more geographically and economically diverse studies need to be undertaken to confirm these initial findings.

\section{References}

1. Coresh J, Astor BC, Greene T, Eknoyan G, Levey AS. Prevalence of chronic kidney disease and decreased kidney function in the adult US population: Third National Health and Nutrition Examination Survey. Am J Kidney Dis 2003;41:1-12.

2. Coresh J, Selvin E, Stevens LA, et al. Prevalence of chronic kidney disease in the United States. JAMA 2007;298:2038-47.

3. Levey AS, Coresh J, Balk E, et al. National Kidney Foundation practice guidelines for chronic kidney disease: evaluation, classification, and stratification. Ann Intern Med 2003;139:137-47.

4. K/DOQI clinical practice guidelines on hypertension and antihypertensive agents in chronic kidney disease. Am J Kidney Dis 2004;43(5 Suppl 1):S1290.

5. Centers for Disease Control and Prevention. Endstage renal disease attributed to diabetes among American Indians/Alaska natives with diabetesUnited States, 1990-1996. JAMA 2001;285:37-8.

6. Buckalew VM Jr, Berg RL, Wang SR, Porush JG, Rauch S, Schulman G. Prevalence of hypertension in 1,795 subjects with chronic renal disease: the modification of diet in renal disease study baseline cohort. Modification of Diet in Renal Disease Study Group. Am J Kidney Dis 1996;28:811-21.

7. Wetterhall SF, Olson DR, DeStefano F, et al. Trends in diabetes and diabetic complications, 1980-1987. Diabetes Care 1992;15:960-7.

8. Agodoa L. African American Study of Kidney Disease and hypertension (AASK)-clinical trial update. Ethn Dis 1998;8:249-53.

9. Go AS, Chertow GM, Fan D, McCulloch CE, Hsu CY. Chronic kidney disease and the risks of death, cardiovascular events, and hospitalization. N Engl J Med 2004;351:1296-305.

10. Anavekar NS, McMurray JJ, Velazquez EJ, et al. Relation between renal dysfunction and cardiovascu- lar outcomes after myocardial infarction. $\mathrm{N}$ Engl J Med 2004;351:1285-95.

11. Chronic kidney disease: making a case for early intervention. Dis Manag Advis 2004;10:13-5.

12. Levey AS, Greene T, Schluchter MD, et al. Glomerular filtration rate measurements in clinical trials. Modification of Diet in Renal Disease Study Group and the Diabetes Control and Complications Trial Research Group. J Am Soc Nephrol 1993;4:1159_ 71.

13. Vassalotti JA, Stevens LA, Levey AS. Testing for chronic kidney disease: a position statement from the National Kidney Foundation. Am J Kidney Dis 2007;50:169-80.

14. Fox CH, Brooks A, Zayas LE, McClellan W, Murray B. Primary care physicians' knowledge and practice patterns in the treatment of chronic kidney disease: an Upstate New York Practice-based Research Network (UNYNET) study. J Am Board Fam Med 2006;19:54-61.

15. Nagykaldi Z, Mold JW, Aspy CB. Practice facilitators: a review of the literature. Fam Med 2005;37: 581-8.

16. Nagykaldi Z, Mold JW, Robinson A, Niebauer L, Ford A. Practice facilitators and practice-based research networks. J Am Board Fam Med 2006;19: 506-10.

17. Soman S, Zasuwa G, Yee J, Soman S, Zasuwa G, Yee J. Automation, decision support, and expert systems in nephrology. Adv Chronic Kidney Dis 2008;15:4255.

18. Denekamp Y. Clinical decision support systems for addressing information needs of physicians. Isr Med Assoc J 2007;9:771-6.

19. National Kidney Foundation. Kidney Disease Outcomes Quality Initiative clinical practice guidelines for chronic kidney disease: evaluation, classification, and stratification. Available at http://www.kidney. org/professionals/kdoqi/guidelines_ckd/toc.htm. Accessed 12 September 2008.

20. Boulware LE, Troll MU, Jaar BG, Myers DI, Powe NR. Identification and referral of patients with progressive CKD: a national study. Am J Kidney Dis 2006;48:192-204.

21. O'Brien MA, Rogers S, Jamtvedt G, et al. Educational outreach visits: effects on professional practice and health care outcomes. Cochrane Database Syst Rev 2007;(4):CD000409.

22. Hartig JR, Allison J. Physician performance improvement: an overview of methodologies. Clin Exp Rheumatol 2007;25(6 Suppl 47):50-4.

23. Jaen CR, Stange KC, Nutting PA. Competing demands of primary care: a model for the delivery of clinical preventive services. J Fam Pract 1994;38: $166-71$.

24. Ostbye T, Yarnall KS, Krause KM, et al. Is there time for management of patients with chronic diseases in primary care? Ann Fam Med 2005;3:209-14. 
25. Kahn LS, Fox CH, Olawaiye A, Servoss TJ, McLean-Plunkett E. Facilitating quality improvement in physician management of comorbid chronic disease in an urban minority practice. J Natl Med Assoc 2007;99:377-83.

26. Macleod U, Mitchell E. Comorbidity in general practice. Practitioner 2005;249:282-4.

27. Brosius FC 3rd, Hostetter TH, Kelepouris E, et al. Detection of chronic kidney disease in patients with or at increased risk of cardiovascular disease: a science advisory from the American Heart Association Kidney And Cardiovascular Disease Council; the Councils on High Blood Pressure Research. Cardiovascular Disease in the Young, and Epidemiology and Prevention; and the Quality of Care and Outcomes Research Interdisciplinary Working Group: developed in collaboration with the National Kidney Foundation. Circulation 2006;114:1083-7.

\section{Appendix 1. PEA Work Tool}

\section{PEA Work tool II}

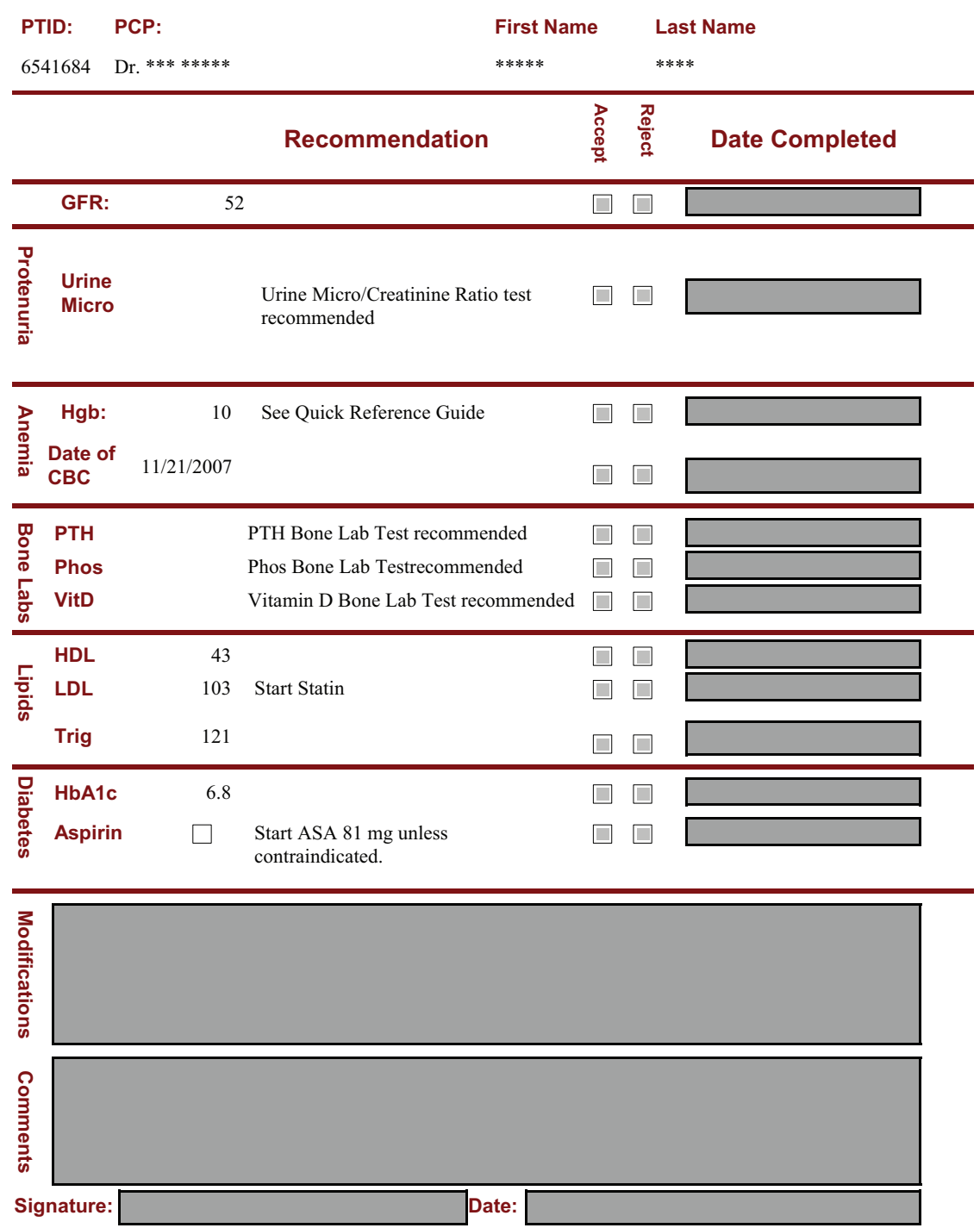

Thursday, January 31, 2008

Page 1 of 1 
Appendix 2. Chronic Kidney Disease Point of Care Guide for the Primary Clinician

Target Patients

- Hypertension

- Diabetes mellitus

- Cardiovascular disease

- Age $>60$

- Family history of CKD

\section{Diagnosis of CKD}

- GFR <60 (not acute situation, lasting >3 months [this is stage $3 \mathrm{CKD}]$ )

- microalbumin/creatinine ratio $>30 \mathrm{mg} / \mathrm{g}$

\section{Eight Actions to Take}

1. Take off unsafe medications

- NSAIDS (caution patient about over the counter meds) and Cox-2 inhibitors.

- Metformin (not dangerous to the kidney but kidney disease increases the chance of lactic acidosis) for GFR $<60$.

- Reduce allopurinol dose to $100 \mathrm{mg} /$ day.

- Do not use bisphosphanates if GFR $<35$.

- Make sure patients going for colonoscopy are not using phosphate preperations like Fleets. This has caused normal people to need dialysis. Go-lytely is a safe alternative.

- Be careful with intravenous contrast.

- Avoid gadolinium if GFR $<30$.

\section{Start ACE or ARB unless contraindicated}

- These are safe and effective at preventing progression of CKD at least up to a GFR of 30 $\mathrm{mL} / \mathrm{min}$ (at this level, a nephrologist should be involved in care).

- There can be long-term clinical improvement despite an initial decrease of $25 \%$ in GFR. A decrease greater than $25 \%$, however, requires a stoppage of ACE/ARB and a work-up for renal artery stenosis.

- Nephrology referral if hyperkalemia occurs. (Particular care must be taken for the patient with $\mathrm{CKD}$ who is on an $\mathrm{ACE}$ and spironolactone, as they may also raise $\mathrm{K}+$ ).
3. Start acetylsalicylic acid $81 \mathrm{mg}$ daily unless contraindicated

4. Get laboratory tests

- Hemoglobin

- Complete metabolic profile (electrolytes, blood urea nitrogen, glucose, creatinine, GFR, aspartate transminase, alanine aminotransferase)

- Calcium, PO4, parathyroid hormone, and 25-OH vitamin D

- HBA1C if the patient has diabetes mellitus

- Fasting lipid profile (total cholesterol, highdenisty lipoprotein, low-density lipoprotein, and triglycerides)

- Urinalysis (looking for hematuria or pyuria); his may be a sign of other forms of kidney disease such as stones or bladder cancer

- Urine microalbumin/creatinine ratio

\section{What to Look for in Laboratory Tests}

- Hemoglobin <12 for men or 13.5 for women; indicates anemia and requires work-up)

- HBA1C >7.0; treat as you would any diabetic but avoid metformin

- high-denisty lipoprotein $<40$

- $\mathrm{Ca}^{++}<8.4$ or $>10.2$

- low-density lipoprotein $>100$ or possibly 70 (CKD is a coronary artery disease equivalent)

- $\mathrm{PO}^{4}>4.6$

- Parathyroid hormone $>70$

- Triglycerides $>150$

- Vitamin D $<30$

- If hemoglobin $<12$ in men or 13.5 in woman, get work-up and treat for anemia

- Get iron/total iron binding capacity (ferritin optional)

- If serum iron/ total iron binding capacity $<20 \%$, patient is iron deficient

- Rule out colon cancer with a colonoscopy

- Replace iron until iron/total iron binding capacity $>20$

- If hemoglobin is $<11$, start erythropoietin

Write a prescription for either darbepoeitin $40 \mu \mathrm{g}$ or erythropoietin 10,000 units sub Q q2 weeks

- Monitor CBC monthly and iron/TIBC every 3 months. Keep iron/TIBC> 20\% 
- Avoid or hold erythropoietin if hemoglobin is $>12.0$

If parathyroid hormone is $>100$ or $\mathrm{PO}^{4}$ is $>4.6$, refer to nephrologist

If $25-\mathrm{OH}$ vitamin $\mathrm{D}$ is $<30$, start ergocalciferol 50,000 units once per month. Recheck calcium, $\mathrm{PO}^{4}$, vitamin $\mathrm{D}$, and parathyroid hormone in 3 months. If vitamin D is very low $(<10)$, treatment can begin weekly.

5. Refer to nephrologist if:

- GFR $<30 \mathrm{~mL} / \mathrm{min}$

- Persistent hematuria should be seen by either an nephrologist or urologist

- Marked proteinuria out of proportion with decreased GFR
- Uncontrolled hypertension

- Recurrent renal calculi

- Parathyroid hormone $>100$ or $\mathrm{PO}^{4}>4.6$

6. Control lipids with statins, fibrates, niacin, and omega-3s

7. Recommend smoking cessation counseling if the patient is a smoker

8. Save and arm and avoid PICC lines if GFR $<45$

- Have all bloods drawn from dominant arm

- Use a single lumen catheter for central access

- This is done to preserve veins for hemodialysis and prevent the need for neck catheters

More people with CKD die of heart attacks than of CKD. 